\title{
Comunicación y crisis del coronavirus en España. Primeras lecciones
}

\section{Communication and coronavirus crisis in Spain. First lessons}

\author{
Carmen Costa-Sánchez; Xosé López-García
}

Cómo citar este artículo:

Costa-Sánchez, Carmen; López-García, Xosé (2020). “Comunicación y crisis del coronavirus en España. Primeras lecciones". El profesional de la información, v. 29, n. 3, e290304.

https://doi.org/10.3145/epi.2020.may.04

Artículo recibido el 24-04-2020

Aceptación definitiva: 03-05-2020

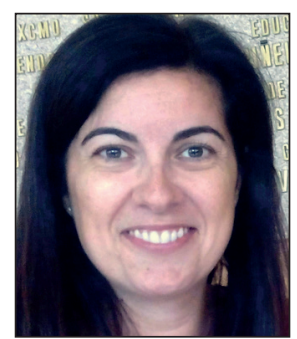

\author{
Carmen Costa-Sánchez $\triangle$ \\ https://orcid.org/0000-0001-8154-9537 \\ Universidade da Coruña \\ Facultade de Ciencias da Comunicación \\ Campus de Elviña, s/n. \\ 15071 A Coruña, España \\ carmen.costa@udc.es
}

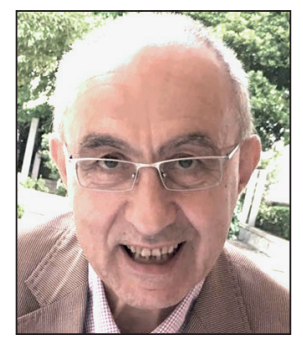

Xosé López-García

https://orcid.org/0000-0002-1873-8260

Universidade de Santiago de Compostela

Facultade de Ciencias da Comunicación

Avenida Castelao, s/n.

15782 Santiago de Compostela, España

xose.lopez.garcia@usc.es

\section{Resumen}

La crisis del coronavirus ha generado una complicada situación a nivel sanitario, económico y social, que requiere de una labor de comunicación fundamental para que la sociedad comprenda y colabore con las medidas adoptadas. A través de la revisión y síntesis de la bibliografía científica y de referencia, el presente trabajo contrapone lecciones aprendidas en crisis de salud pública previas con la comunicación efectuada desde las voces institucionales y desde la cobertura de prensa durante las primeras etapas de la crisis del Covid-19 en España. Se detecta que la ausencia de un anuncio temprano (o adelantamiento del escenario negativo) y los mensajes de control en un contexto de incertidumbre provocaron una transición brusca a la siguiente etapa de la crisis, coincidente con una explosión en el número de contagios, lo que generó miedo. La fórmula de portavocía adoptada incumple toda recomendación de comunicación de crisis. En cuanto a la cobertura periodística, se localizan ejemplos de alarmismo y de sensacionalismo en el tratamiento informativo de la pandemia. Los datos numéricos han sido el recurso informativo más valioso y las infografías el contenido multimedia más desarrollado. Dado que la pandemia se encuentra todavía en curso, se recomienda el aprendizaje y la corrección de la comunicación e información a los ciudadanos en base a este conjunto de primeras lecciones en las etapas siguientes o futuras crisis.

\section{Palabras clave}

Coronavirus; Covid-19; Pandemias; Crisis de salud pública; Crisis sanitarias; Comunicación de crisis; Comunicación de riesgos; Comunicación institucional; Salud; Prensa; Cobertura periodística; Comunicación científica.

\footnotetext{
Abstract

Coronavirus crisis has caused a complicated situation at a sanitary, economic and social levels, that requires of a fundamental work in communication area so that the society comprise and collaborate with the adopted measures. Through the review and synthesis of the scientific and reference literature, the present paper contrasts lessons learnt in previous public health crisis against the communication implemented from the institutional voices and from the press coverage during the first stages of the Covid-19 outbreak in Spain. This paper detects that the absence of an early announcement (or overtaking of the negative stage) and the control messages in a context of uncertainty caused a brusque transition to the following stage of the crisis, coincident with an explosion of the number of contagions, what generated fear. The spokesperson formula applied is against all recommendations of crisis communication. Regarding the journalistic coverage, examples of alarmism and sensationalism news arised. The numeric data have been the resource more valuable and the computer graphics the multimedia content more developed. Since the pandemic is still on course, it is recommended the learning and the adjustment of the communication and information to the citizens in the following stages of this crisis or in future crisis taking into account the group of first lessons presented here.
} 


\section{Keywords}

Coronavirus; Covid-19; Pandemics; Public health crisis; Health crisis; Crisis communication; Risk Communication; Institutional communication; Health; Press; Press coverage; Scientific communication.

\section{Introducción. Crisis de salud pública y comunicación}

La Organización Mundial de la Salud en el marco del Sixth Futures Forum on Crisis Communication (2004) recoge:

"En salud, crisis y comunicación están íntimamente relacionadas. Todas las crisis de salud son también crisis de comunicación".

La necesidad de explicar a la ciudadanía lo que está sucediendo y cuáles son los riesgos, también de implicarla en la solución, convierten a la comunicación en una importante aliada de la gestión política, social, institucional y sanitaria de cualquier situación de este tipo.

Las crisis de salud pública han sido definidas como

"eventos no planeados, que implican una amenaza real, percibida o posible contra el bienestar de la sociedad (o algún segmento), el ambiente o una agencia de salud afectada" (Churchill, 2000, p. 125).

La percepción de esa amenaza o de ese riesgo es un factor de gran importancia. No es lo mismo el riesgo objetivo, que el percibido. En la fórmula de Sandman (2012), el riesgo percibido es igual a la suma de la amenaza y la indignación (hazard + outrage). En la crisis del coronavirus, asistimos a una situación que genera una amenaza elevada con elevada indignación. Además, dicha percepción se acentúa si el tipo de riesgo para la salud de la población cumple las siguientes características (Sandman, 2012):

- es involuntario más que voluntario;

- se reparte de forma desigual entre la población;

- no se puede evitar aunque se tomen las precauciones necesarias;

- procede de fuentes no familiares o novedosas;

- causa un daño irreversible y oculto;

- supone peligro para los niños o mujeres embarazadas o para las futuras generaciones;

- daña a personas conocidas más que a víctimas anónimas;

- es desconocido desde el punto de vista científico o incluso si es protagonista de afirmaciones contradictorias procedentes de fuentes responsables (o incluso peor, desde la misma fuente);

- causa la muerte.

Los riesgos generados por el coronavirus cumplen la mayoría de estos requisitos, lo que predice que la fórmula de Sandman alcanza, al menos en España, una percepción de la situación muy marcada en términos de miedo y tristeza para la sociedad (Brennan, 2014).

Según la WHO (2013), la Ciencia reconoce cierto grado de incertidumbre sobre muchos riesgos y la magnitud de otros no se conoce en profundidad. Además, no todas las personas afrontan el mismo nivel de riesgo, que puede estar desigualmente distribuido entre la población. Ello hace que la comunicación en estas situaciones sea compleja y requiera el manejo también difícil de variables como información puntual y correcta, empatía, esperanza, confianza pública en las autoridades y políticas efectivas.

En un contexto de globalización, se globalizan las empresas, los movimientos políticos o ideológicos, pero también los riesgos para la salud. En la sociedad del riesgo global (Beck, 2002), aparecen nuevas amenazas para la salud de los individuos. La combinación de cambios en los modos de consumo, los estilos de vida, la globalización, la extensión de los viajes y los movimientos migratorios de millones de personas han ayudado a incrementar los riesgos para la salud (Mahy et al., 2017). Dichas amenazas se caracterizan por la expansión (internacionalización) de los riesgos y por el nivel de alarma que generan en poblaciones y gobiernos.

La última vez que la OMS declaró la categoría de pandemia fue en 2009 debido al brote de gripe A H1N1. Este tipo de influenza fue identificado por primera vez en Estados Unidos y se propagó rápidamente por el mundo. Este nuevo virus H1N1 contenía una combinación exclusiva de genes de virus de influenza que nunca antes había sido identificada en personas o animales. Durante el primer año de la pandemia (2009-2010), se estima que entre el 0 y el 0,01 por ciento de la población mundial falleció debido a complicaciones respiratorias relacionadas con el virus (CDC, 2020). Según explica Esparza (2016), en los últimos años han ocurrido 11 epidemias o pandemias de importancia a nivel mundial y con excepción de dos epidemias de cólera en Zimbabue y en Haití, todas las demás han sido causadas por virus. Éstas incluyen epidemias de SARS (síndrome respiratorio agudo grave), chikungunya, influenza porcina A-H1N1, sarampión (en la República Popular del Congo), MERS (síndrome respiratorio de Oriente Medio), Ébola y Zika. Dos de esos virus (SARS y MERS) pertenecen a la familia de los coronavirus, que también incluye a otros virus que causan enfermedades respiratorias y que son transmitidos por secreciones respiratorias. En España, la crisis más reciente ha sido un brote puntual y aislado generado por el primer contagio de ébola a una profesional sanitaria en octubre de 2014. 
A pesar de que en los siglos XX y XXI no se había asistido a una situación de tal magnitud (por la cantidad de países y población afectada) respecto a la existente en la actualidad, lecciones extraídas de experiencias previas han arrojado una serie de análisis y recomendaciones a la hora de plantear la gestión y la comunicación con los ciudadanos. La primera de ellas, acerca de la necesaria preparación ante una posible situación de este tipo.

"Las crisis de salud pueden afectar potencialmente a una gran proporción de la población y pueden conducir a un aumento significativo de la mortalidad o a una tasa de mortalidad anormalmente alta. Esto debería integrarse en el concepto general de vigilancia nacional e internacional para proporcionar una respuesta preparada en caso de crisis" (Mahy et al., 2017).

La segunda, sobre la importancia de la comunicación como un factor determinante dentro de la gestión. Según los expertos reunidos en el Sixth Communication Forum (Scarcella et al., 2013; WHO, 2004), que participaron en la gestión de crisis como la de las dioxinas en Bélgica, la alerta de gripe aviar en Austria, el SARS en Noruega y en Ontario, en la comunicación de crisis de salud pública, deben aplicarse una serie de principios como los siguientes:

1. Ser pro-activos e iniciar la comunicación pública tan pronto como sea posible al inicio de la crisis, puesto que la información tiene un efecto calmante, incluso si trata sobre riesgos para la salud.

2. Ser accesible para la prensa y comunicar con regularidad, pero evitar la saturación informativa. Las conferencias de prensa diarias deben reservarse sólo para los momentos punta de la crisis.

3. Comunicar información sobre riesgos para la salud con consejos para el público sobre cómo reaccionar por ellos mismos a esas amenazas.

4. Comunicar de manera sencilla, precisa, transparente, sin ser condescendiente y en el debido momento, antes de que la acción sea emprendida.

5. Ser honesto y evitar mantener información secreta para tranquilizar al público.

6. Ser claro sobre lo que uno sabe y sobre lo que no sabe (todavía).

7. No sentirse inferior, al admitir que falta información o evidencias.

8. Asegurar coherencia en los mensajes comunicados a la prensa por las autoridades sanitarias (buscando el efecto de una sola voz).

9. Presentar las cifras correctas para una presentación precisa y una acción concreta.

10. Mostrar respeto y empatía con la ansiedad del público y comunicar de manera acorde.

Además, se necesita mayor investigación para examinar crisis que duran meses en lugar de días o semanas y así ayudar a los comunicadores a determinar cómo adaptar las estrategias y tácticas a lo largo del tiempo (De-Giusti; Manochi, 2012). A nivel europeo, se precisa de una mayor conexión entre la investigación académica y la práctica (ECDC, 2013).

En España, el primer caso de coronavirus a nivel local se dio a conocer el 31 de enero de 2020. Se trataba de un paciente alemán ingresado en estado leve en la isla de La Gomera. El 24 de febrero se detectaban los primeros casos en la Comunidad de Madrid, Cataluña y la Comunidad Valenciana. A 20 de abril de 2020, hay cuantificados en España, 200.210 casos totales, 20.852 fallecidos y 80.587 recuperados (Ministerio de Sanidad, 2020). El objeto de este trabajo no es evaluar la gestión de la crisis en términos sanitarios (ni en otros en los que ha tenido y está teniendo repercusión), sino poner el foco en la comunicación efectuada, atendiendo a una doble perspectiva: fuentes institucionales y cobertura de prensa.

\section{Metodología}

La presente investigación pretende realizar una revisión de la bibliografía científica sobre crisis de salud pública y comunicación para hacer acopio de las principales conclusiones/recomendaciones extraídas de análisis previos. La naturaleza de la Ciencia es y debe ser acumulativa, de manera que se construye sobre conocimiento ya aportado, que se contrasta, se interroga o se respalda en cada nuevo resultado obtenido.

El objetivo de este análisis es realizar una recopilación sobre lecciones aprendidas con anterioridad y aproximar sus principales conclusiones a aspectos de la comunicación y cobertura periodística de la actual crisis del coronavirus. Los dos principales ejes que articulan el análisis son, por tanto, el de la comunicación de las voces oficiales y el de la cobertura de prensa. En un segundo momento, dicha recopilación se pone en contraste con aspectos que ya pueden observarse en la comunicación que están recibiendo los ciudadanos tanto de las autoridades gubernamentales como de la prensa. Tal y como indican Gérvas y Hernández-Aguado (2009), las crisis son retos de los que debemos aprender, tanto de los errores como de los éxitos, pues sirven para poner a punto los dispositivos y perfeccionar la respuesta ante otras próximas, inevitables e impensables. La Organización Mundial de la Salud reconoce la importancia y los beneficios de ese aprendizaje previo (WHO, 2020).

"Cada emergencia de salud pública enfrenta nuevos desafíos de comunicación y puede beneficiarse de las lecciones aprendidas previamente. El brote de Covid-19 desafía los sistemas de salud pública y su capacidad para comunicarse de manera efectiva con sus poblaciones. La falta de comunicación adecuada conduce a una pérdida de confianza y reputación, impactos económicos y, en el peor de los casos, pérdida de vidas" (WHO, 2020, p. 1). 
La correspondiente revisión de la bibliografía científica no pretende por lo tanto establecer un estado de la cuestión (de lo que se ha teorizado sobre comunicación de crisis y de riesgos hasta el momento), sino tomar aspectos concretos derivados del análisis de anteriores experiencias. Para ello, se llevó a cabo una búsqueda de artículos sobre crisis de salud y comunicación publicados en revistas indexadas en Scopus en el período 2010-2020. En el proceso de rastreo, se localizaron artículos científicos que contuviesen en su título, resumen o palabras clave los términos, tanto en castellano como en inglés, "crisis de salud", "crisis de salud pública" y "comunicación”. Del total de artículos localizados (163), además de eliminar las duplicaciones de textos surgidas de la búsqueda en castellano-inglés, se eliminaron manualmente aquellos trabajos que no se ajustaban a la temática objeto de estudio (artículos sobre manejo de episodios de crisis psiquiátricas, desde distintos enfoques, o aquellos sobre gestión sanitaria o aspectos biomédicos de distintas enfermedades, también sobre la cobertura y la gestión de la comunicación de otro tipo de crisis relacionadas con la salubridad de la industria hotelera, por ejemplo), obteniendo un total de 102 artículos científicos. Tras la revisión de todos ellos, se sintetizan en este texto aquellos con implicaciones prácticas para la comunicación de la pandemia actual.

También se realizó una búsqueda en PubMed, base de datos especializada en ciencias de la salud, con iguales términos y para el mismo período, obteniendo un total de 57 resultados. El índice de coincidencia con respecto a la búsqueda previa fue muy elevado, de modo que tan solo se añadieron a la revisión realizada cuatro trabajos nuevos de interés.

Asimismo, se implementó una búsqueda complementaria en Google Scholar para completar la revisión de la bibliografía científica con otros posibles trabajos previos de interés publicados en plataformas no indexadas en las bases de datos ya indicadas. La búsqueda (con los mismos términos y período de tiempo) generó 451 resultados, de los que 71 respondían al objeto de estudio con potencial adecuación, aunque se seleccionaron aquellos con implicaciones prácticas y complementarias para la síntesis de las lecciones previas.

En un segundo momento, se contrapuso dicho conjunto de aportaciones con lo sucedido a lo largo de este primer período (mes de marzo y hasta el 20 de abril, fundamentalmente) en la crisis del coronavirus, tomando dicha información de diversas fuentes, fundamentalmente, voces oficiales del Gobierno de España y cibermedios a nivel nacional.

El presente trabajo pretende realizar una recopilación sobre lecciones aprendidas en situaciones previas y aproximarlas a aspectos de gestión de comunicación y cobertura periodística de la actual crisis del coronavirus en España. Los dos principales ejes que articulan el análisis son, por lo tanto, el de la comunicación de las voces oficiales y el de los cibermedios de referencia.

\section{Resultados}

\subsection{Lecciones desde la comunicación institucional}

La importancia del anuncio temprano de una situación de estas características ha sido determinada en estudios previos, así como en las recomendaciones de la WHO (2005). En el estudio de Jones et al. (2010) se sugiere que si se le plantea a la sociedad el peor escenario posible cuando

\section{La importancia del anuncio temprano de una situación de estas características es una de las recomendaciones básicas de la $\mathrm{WHO}$} solo existe el riesgo hipotético de que se produzca, asumirán dicho riesgo de manera menos peligrosa y serán menos proclives a entrar en pánico cuando la pandemia suceda. Por tanto, en los estadios previos de la pandemia (antes de que se identifiquen casos en un país determinado), los mensajes deberían estar focalizados en incrementar el conocimiento de la enfermedad y dar a conocer sencillos comportamientos protectores para reducir el riesgo de transmisión.

De hecho, trabajos previos (Jang; Park, 2018; Vos; Buckner, 2015) parten del modelo de Crisis and Emergency Risk Communication (CERC) CDC (2020), que establece cinco etapas con diferentes tipos de mensajes asociados a cada una de ellas (Reynolds; Seeger, 2005).

- En la primera etapa (precrisis), de advertencia y preparación, la comunicación debe orientarse al conocimiento del riesgo por parte de la población, a su preparación para un posible evento adverso, al desarrollo de recomendaciones consensuadas y a fomentar cambios comportamentales que reduzcan la probabilidad del daño.

- En la segunda etapa (evento inicial) una comunicación rápida debe establecer empatía, seguridad y reducción de la agitación emocional, así como el entendimiento general de las circunstancias de la crisis, sus consecuencias, posibles resultados basados en la información disponible y de las actividades de respuesta personal y de autoeficacia.

- En la tercera etapa (mantenimiento) se necesita un entendimiento público más preciso de los riesgos, así como de los antecedentes y elementos de fondo, obtener feedback de los públicos afectados y corregir desinformaciones y rumores, explicación y reiteración de la autoeficacia y las actividades personales de respuesta y toma de decisiones informada por parte del público fundamentada en el entendimiento tanto de los riesgos como de los beneficios.

- La cuarta etapa (resolución) necesita comunicar de cara a informar y persuadir de los esfuerzos de recuperación y de reconstrucción, facilitar una discusión abierta y resolución de asuntos relacionados con la causa, la culpabilidad, la responsabilidad y la adecuación de la respuesta, generar el entendimiento público de los nuevos riesgos y nuevos entendimientos del riesgo.

- En la quinta (evaluación) se requiere documentar, formalizar y comunicar las lecciones aprendidas y determinar acciones específicas para mejorar la comunicación de crisis y la capacidad de respuesta ante una crisis. 
A lo largo de este marco, los mensajes dirigidos a la población deben permitir dotar de sentido a lo que acontece (colocar los acontecimientos en marcos; comprender qué ha sucedido; acomodar lo inesperado; interactuar para producir entendimiento e identificar modelos), así como ofrecer información eficaz, es decir, que le ayude a dar una respuesta adecuada. Información sobre la probabilidad de que algo suceda en el futuro y mensajes sobre la evolución en torno a la crisis (Vos; Buckner, 2015). Los mensajes de eficacia contribuyen a una respuesta efectiva educando a la gente para que responda adecuadamente. Los mensajes altamente instructivos generan tanto conocimiento como sensación de eficacia (Frisby, 2014; Rodríguez Andrés, 2011).

Acerca de la actitud a desarrollar por las autoridades sanitarias en situaciones de este tipo, la transparencia, la información puntual y la sensibilidad y empatía hacia la población son algunas de las claves que se manejan en la bibliografía científica previa (Savoia; Lin; Viswanath, 2013). La transparencia implica ni minimizar ni exagerar el nivel de riesgo, discutir la incertidumbre de datos e información, sus fortalezas y sus debilidades, reconocer aquello que se desconoce o sobre lo que no se tienen seguridades, si se han producido errores, corregirlos rápidamente y si la información evoluciona o se encuentra incompleta, enfatizar las reservas sobre su fiabilidad (Covello, 2001).

La transparencia es además una pieza esencial si el público debe confiar en las autoridades encargadas de gestionar la amenaza, pues sin esa confianza, será muy difí-

\section{La transparencia es una pieza esencial} cil convencer a la gente de que adopte comportamientos necesarios de cara a tener ese riesgo bajo control. La falta de transparencia incrementa la probabilidad de rumores y desinformación y entorpece el control de la enfermedad (WHO, 2008).

"La confianza es la base de la comunicación. Mantener una actitud abierta y honesta e involucrar activamente a las partes interesadas para abordar sus necesidades de información sobre riesgos servirá para generar confianza y facilitar colaboraciones multisectoriales para enfrentar una crisis de salud pública" (Qiu et al., 2018, p. 587).

La actitud de los agentes gubernamentales debe ser dialógica, pues una ausencia de competencia dialógica disminuiría la credibilidad de la información proporcionada en relación a los riesgos (Yang, 2018). Debido a la incertidumbre y contingencia del riesgo, la comunicación de riesgos en crisis de salud pública necesita seguir los principios de apertura y transparencia (Qiu; Chu, 2019). Debe buscarse, activamente, además, el apoyo de la sociedad, pues las autoridades sanitarias solo pueden asegurar la eficacia de las medidas de cuarentena durante una epidemia emergente por medio de la colaboración con las comunidades concernidas y con los ciudadanos y a través de una cuidadosa guía para la participación pública (Ding, 2014).

En lo referente a los canales, aunque se anima a las administraciones pertinentes a la adopción del uso de las redes sociales en la comunicación de la crisis, se subraya que es necesario seguir utilizando los medios de comunicación tradicionales para aquella población que no emplea las nuevas plataformas digitales. De este modo, se consideran las redes sociales como canales de comunicación complementarios a los medios tradicionales (Takahashi; Tandoc; Carmichael, 2015). De hecho, la televisión y la prensa han sido identificados previamente como los dos medios informativos de referencia en otras crisis sanitarias (Park; Boatwright; Johnson, 2019; Mondragón, 2016).

En cuanto al aspecto específico de la portavocía, estudios previos apuntan a la conveniencia de un solo portavoz (Mestre-Ortega et al., 2018; Calleja-Reina; Becerra, 2017; Jurado; Jurado, 2014) o una portavocía muy coordinada y con una sola voz (WHO 2005). A propósito de la crisis del ébola, el Centro de Estudios de Ciencia, Comunicación y Sociedad (2014) apuntaba que el portavoz ideal sería una persona que tuviera a la vez el conocimiento y la autoridad. No es suficiente alguien que sepa mucho sobre un tema pero que no tenga autoridad para asegurar que se hará lo correcto. Tampoco sirve que un cargo de alta autoridad no sepa reaccionar ni dar respuestas ante situaciones socialmente complicadas, pues genera desconfianza y alarma en la sociedad (Centro de Estudios de Ciencia, Comunicación y Sociedad, 2014). Su credibilidad es un factor esencial para cualquier portavoz, especialmente en crisis sanitarias (WHO, 2005).

Algunas de estas lecciones pueden mirarse ya en el espejo de la actual crisis del coronavirus en España.

Las referencias localizadas indican que el mensaje institucional, en la etapa de precrisis, cuando todavía no se habían detectado casos de Covid-19 a nivel interno, era de que no había que tener percepción de riesgo y de que la incidencia de la enfermedad, en caso de haberla, resultaría baja y controlada.

Mensajes en este sentido fueron:

"La población tiene que tener un nivel de percepción de riesgo muy bajo. Si vienen de Wuhan y tienen síntomas, que vayan al médico tranquilamente. Pero estamos en época de gripe: cualquier sintomatología, incluso si vienen de allí, lo más probable es que sea gripe" (Fernando Simón, El país, 25/01/2020)

o

“Nosotros creemos que España no va a tener como mucho más allá de algún caso diagnosticado. Esperemos que no haya transmisión local. Si la hay, será una transmisión muy limitada y muy controlada" (Fernando Simón, El español, 30/03/2020). 
De hecho, la comparación con la gripe funcionó como mecanismo para relativizar (restar) la importancia del Covid-19 y tranquilizar a la población, en lugar de ir avanzando los mensajes de activación de escenarios negativos y/o mensajes de eficacia para promover comportamientos responsables.

"La gripe es un problema conocido, llevamos muchos siglos con ella, pero la gente no se agobia con la gripe porque es algo a lo que nos hemos acostumbrado y no podemos quitarnos de encima. Me sorprende este exceso de preocupación" (Fernando Simón, La sexta, 16/02/2020).

Los mensajes, en síntesis, de esta primera etapa (precrisis e, incluso inicial, cuando se detectan los primeros casos), apuntan a un exceso de tranquilización de la población, en lugar de adelantar un posible escenario negativo, en la línea de lo ya explicado. Cuando comienzan a suceder los primeros casos, el mensaje principal continúa siendo de control y seguridad, de ahí que, cuando el incremento de casos es mayúsculo, el cambio de mensaje resulte radical (ver tabla 1). El hecho de tener que alertar a la población, frente a la tranquilización de la inmediata etapa previa, genera que ésta experimente la sensación de miedo, se abastezca masivamente en el supermercado

(“Los rasgos psicológicos de las compras compulsivas por el coronavirus", El confidencial, 10/03/2020)

y siga con atención pero intranquilidad las medidas que se le sugieren de aislamiento social y de prevención (por ejemplo, el lavado de manos).

Con respecto a la fórmula de portavocía, en la crisis del coronavirus en España, se localizan tres etapas fundamentalmente:

a) La primera etapa (precrisis), sin casos locales, en la que el portavoz único es el director del Centro de Coordinación de Alertas y Emergencias, Fernando Simón;

b) La segunda etapa, con casos locales (inicio), en la que sigue como portavoz principal Fernando Simón, complementando su labor dos portavoces institucionales (el ministro de Sanidad y el presidente del Gobierno);

c) Una tercera etapa (mantenimiento), en la que comparecen los integrantes técnicos del Comité técnico de gestión del coronavirus, pero también el presidente del Gobierno y los ministros aludidos después del Consejo de Ministros correspondiente, además de los propios ministros del Comité técnico, conformado por el Ministerio de Sanidad, el Ministerio de Defensa, el del Interior y el de Transportes. A día 05 de abril se contabilizaba un total de 53 comparecencias, una media de dos/día desde el 12 de marzo, siendo solo cuatro los miembros del Gobierno que no habrían intervenido en ruedas de prensa oficiales (La voz de Galicia, 05/04/2020). A día 20 de abril, se contabilizan 91 desde el 12 de marzo sin contar las intervenciones en el Congreso (La voz de Galicia, 20/04/2020).

Tabla 1. Mensajes y portavoces en las etapas de evolución de la crisis.

\begin{tabular}{|c|c|c|c|}
\hline & Precrisis & Inicio & Mantenimiento \\
\hline Portavoz/es & $\begin{array}{l}\text { - Fernando Simón, Director del Centro } \\
\text { de Coordinación de Emergencias y } \\
\text { Alertas sanitarias. }\end{array}$ & $\begin{array}{l}\text { - Fernando Simón, Director del Centro } \\
\text { de Coordinación de Emergencias y } \\
\text { Alertas sanitarias. } \\
\text { - Ministro de Sanidad. } \\
\text { - Presidente del Gobierno. }\end{array}$ & $\begin{array}{l}\text { - Portavoces Comité Técnico Gestión } \\
\text { del Coronavirus. } \\
\text { - Portavoces institucionales. }\end{array}$ \\
\hline $\begin{array}{l}\text { Mensajes } \\
\text { clave }\end{array}$ & $\begin{array}{l}\text { - Tranquilidad } \\
\text { - } \text { Control } \\
\text { - Riesgo bajo }\end{array}$ & $\begin{array}{l}\text { - Tranquilidad } \\
\text { - Control } \\
\text { - Confianza Sistema Nacional Salud } \\
\text { - Riesgo moderado }\end{array}$ & $\begin{array}{l}\text { - Guerra } \\
\text { - Alerta } \\
\text { - Unión } \\
\text { - Agradecimiento } \\
\text { - Empatía } \\
\text { - Mensajes autoeficacia } \\
\text { - Adelanto de escenarios negativos } \\
\text { - Incertidumbre } \\
\text { - Riesgo alto }\end{array}$ \\
\hline
\end{tabular}

En este sentido, habría que hablar de una multiplicación de portavoces, lo que generó a su vez, necesariamente, una elevada y detallada cantidad diaria de información sobre las cuestiones sanitarias y no sanitarias relacionadas con la crisis. También ha dado lugar a contradicciones en el mensaje institucional:

("Tormenta política al declarar un general que la Guardia Civil trabaja para minimizar bulos contrarios al Gobierno", El país, 20/04/2020).

Atendiendo al nivel jerárquico de la fuente, se garantiza de este modo el mantenimiento del liderazgo informativo diario y una gestión proactiva de la comunicación de la pandemia.

Sin embargo, una mayor exigencia de transparencia por parte de periodistas y medios de comunicación después de las primeras semanas ha generado críticas a los portavoces oficiales, tanto en lo referente a la forma de canalizar las preguntas tras las ruedas de prensa correspondientes, como en lo relativo al detalle de los datos facilitados.

Sobre la primera cuestión, el manifiesto 'La libertad de preguntar' recogía la crítica de más de trescientos periodistas de múltiples medios de comunicación que consideraban que el filtrado de preguntas después de las ruedas de prensa por 
medio del secretario de Estado de Comunicación revelaba censura e interés por el control de la información:

(“La prensa española se queja de las preguntas telemáticas al Gobierno por la pandemia”, EFE, 01/04/2020).

Finalmente, tras varias propuestas de las asociaciones de prensa, desde el 06 de abril se puso en marcha un nuevo sistema, en el que se permiten preguntas en directo, se divide a los medios en dos grandes grupos (medios habituales $y$ no habituales) que disponen en total de diez turnos que se organizan de forma aleatoria en uno de los casos y rotatoria en el otro:

(“El nuevo sistema de preguntas en directo de Moncloa: un sorteo y turnos rotatorios para los medios", El diario. es, 06/04/2020).

En lo relativo a los datos, el Gobierno ofrece diariamente un informe de datos de casos confirmados y de fallecimientos, pero no sobre la ocupación de las unidades de cuidados intensivos (UCls), ni el desglose por áreas de salud o municipios, ni por sexo-edad, ni sobre los tests -pruebas PCR realizadas-:

("Los datos del brote de coronavirus que el Gobierno no publica", Maldita.es, 02/04/2020).

Debe apuntarse también que los datos del sistema de monitorización de mortalidad (MoMo, sistema para la vigilancia de la mortalidad diaria por todas las causas en España, gestionado por el Centro Nacional de Epidemiología) no han formado parte del discurso oficial, a pesar de que su objetivo es identificar patrones inusuales de mortalidad y estimar el impacto en la mortalidad de la población de cualquier evento de importancia para guiar la acción en Salud Pública

Los datos y la forma de contabilizar infectados y decesos continúan generando discrepancias (además de polémica en la arena política) hasta el punto de que a día 17 de abril el Ministerio de Sanidad comunicó que debía rehacer la serie histórica de datos y publicó unos criterios unificados para la aplicación de un sistema similar por parte de las Comunidades Autónomas:

("Sanidad se ve obligada a corregir la serie histórica ante las discrepancias en los datos sobre coronavirus", El país, 17/04/2020).

\subsection{Lecciones desde la cobertura mediática}

Se puede afirmar que, debido al interés que las crisis sanitarias generan en la sociedad, los medios dedican mucho espacio de su temario a esta temática. A pesar de que el modelo general apunta a un interés intensivo por parte de la prensa internacional durante el primer mes desde la declaración de una emergencia de salud pública, y un decaimiento a partir de ahí, también se detecta que la cobertura periodística se incrementa a partir de la detección del primer caso interno, dentro del país (Smith et al., 2013).

Las investigaciones previas sobre la cobertura de prensa de la gripe A han evidenciado un alarmismo elevado. Tanto la prensa de calidad como la sensacionalista amplificaron los riesgos del virus $\mathrm{H} 1 \mathrm{~N} 1$, enfatizando el conflicto y el daño, presentando la información de una manera más dramatizada y usando marcos amplificadores del riesgo, aunque la prensa tabloide exhibió un mayor énfasis en la emoción a la hora de presentar la información (Rossman; Meyer; Schulz, 2018). El relato, además, se estableció en términos de amenaza para la vida y de una guerra en la que el virus tenía ventaja sobre los humanos (Gadekar, 2014).

En la prensa española, también se evidencia el alarmismo de dicha cobertura informativa, fundamentalmente, en los primeros meses de su llegada a España, por varios motivos, entre ellos, la comparación con crisis sanitarias anteriores como la pandemia de gripe de 1918. La comparación con este virus fue, probablemente, una de las causas de la alarma social y mediática surgida al inicio de la gripe A (García-Frontiñán, 2017).

Además, se apunta que el alarmismo estuvo acompañado de cierto sensacionalismo puesto que se difundieron datos personales y clínicos de algunos afectados por la enfermedad, por ejemplo, relativos a la primera víctima mortal de la enfermedad en España. Los medios de comunicación tampoco respetaron en todo momento las normas de confidencialidad de la historia clínica de los pacientes y de su derecho a la intimidad (García-Frontiñán, 2017).

Según concluye Lorente (2010), además de la actuación alarmista y catastrofista en relación con el tratamiento informativo de la actualidad de la gripe A, marcado por la producción de expectativas catastrofistas y la espectacularización de los efectos potenciales de la enfermedad, el componente gráfico de estas informaciones era inadecuado, pues enfatizaba los escenarios hipertecnificados de la investigación y el control sanitario, confrontados con las tragedias cotidianas o los comportamientos meramente anecdóticos para el afrontamiento ciudadano del riesgo sanitario.

El análisis de la cobertura periodística del ébola coincide en el alarmismo (Monjas; Gil-Torres, 2016) y el carácter sensacionalista del tratamiento informativo, tanto por la relevancia de la imagen, como por la personalización de la información, la búsqueda de fuentes alternativas y el léxico alarmista. Especialmente llamativa es la utilización de imágenes de la afectada, su marido y su perro por parte de todos los diarios nacionales (Gou, 2017). Los motivos que han dado lugar a más infografías han sido los aparentemente más insignificantes. Cómo ponerse y quitarse el traje de protección son instrucciones que casi ninguno de los lectores necesitará (Centro de Estudios de Ciencia, Comunicación y Sociedad, 2014).

En cuanto a la cobertura en prensa del Covid-19, ha generado una atención creciente por parte de la prensa española, 
desde la etapa de precrisis al momento actual, hasta el punto de que el temario de la prensa ya focaliza toda su atención en la información y el análisis sobre el coronavirus y sus consecuencias.

Del contraste con lo analizado, se observa que la comparativa con la gripe de 1918 ha sido recurrente por distintas cabeceras de referencia:

- "Coronavirus: mensajes de la gripe de 1918", La vanguardia, 23/02/2020;

- "Estos son los espeluznantes paralelismos de la gripe de 1918 con el Covid-19", El confidencial, 29/03/2020.

El lenguaje de guerra se ha incorporado a la redacción de los titulares:

- "Así es el cuartel general de la lucha contra el virus por territorios", La vanguardia, 20/03/2020.

La metáfora del virus como asesino, aunque en menor medida, también se ha utilizado:

- "Coronavirus: Anatomía de un asesino", La razón, 15/03/2020;

- "Fotografían al coronavirus: el verdadero rostro del asesino", XLSemanal, 08/04/2020.

Todos ellos ingredientes no recomendados en las guías para informar sin generar alarma o miedo (Asociación Española de Comunicación Científica, 2020).

Además, las informaciones han estado muy pendientes del dato, de la cifra, lo que ha propiciado un esfuerzo de creación de infografías que ayudasen a entender y a visualizar la evolución del virus en comparación con otros ámbitos o territorios (tanto a nivel internacional como internamente). Los infográficos, en este sentido, se han convertido en el recurso estrella de apoyo a la información, sobre todo en forma de gráficos de evolución, líneas temporales, gráficos de barras/ columnas, tablas y mapas interactivos de visualización. Datos + información/ interpretación ha sido la fórmula destacada de esta cobertura periodística tanto en medios matriciales, como en nativos digitales, a nivel nacional e internacional, como:

- "7 Ways to explore the math of the coronavirus using The New York Times", TNYT, 02/04/2020);

- "Coronavirus world map: which countries have the most cases and deaths", The guardian, 20/04/2020;

- "Coronavirus: visualisez le bilan des morts du Covid-19, qui dépasse largement celui du SRAS", Le monde, 05/02/2020;

- "Covid-19: ultrapassada a barreira de un milhao de casos, Público, 03/04/2020.

Además de la propuesta infográfica, también se han generado algunos reportajes multimedia (puntualmente, a nivel España) de interés para la sociedad, como los de La vanguardia (Imagen 1) para explicar cómo proceder a la vuelta del supermercado

(“¿Qué debo hacer cuando regreso del supermercado?”, La vanguardia, 01/04/2020)

o en qué se diferencia el virus de la gripe

(“Por qué la Covid-19 no es como una gripe", La vanguardia, 15/04/2020).

O para explicar a los ciudadanos cómo el confinamiento frena la capacidad de contagio

(“Cómo el confinamiento frena la cadena de contagios", El país, 04/04/2020).

En el empleo de vídeo, por habitual, destaca el vídeo en directo ligado a la cobertura de las conferencias de prensa de los portavoces oficiales. Como aspecto más novedoso, puede indicarse este vídeo 3D producido por El país para mostrar cómo afecta el virus a los pulmones:

(“Así ataca el coronavirus en los casos más letales", El país, 10/04/2020).

Sobre el uso de imágenes, la fotografía de un fallecido rodeado por los sanitarios con los trajes de protección en una reciente portada del diario El Mundo ha sido la que más controversia ha generado por su sensacionalismo:

(“La crisis se cebará con España por la gestión del coronavirus", El mundo, 15/04/2020).

Los esfuerzos también han apostado por la lucha contra la desinformación y los bulos, mediante programas de fact-checking. La circulación de noticias falsas (sobre el origen del virus, su tratamiento, el intento de control de la información, $B O E$ falso, imágenes falsas, vinculación con la tecnología 5G, etc.) han proliferado, lo que ha conducido a la denominación de 'infodemia'

("La pandemia se convierte en infodemia", El país, 19/04/2020).

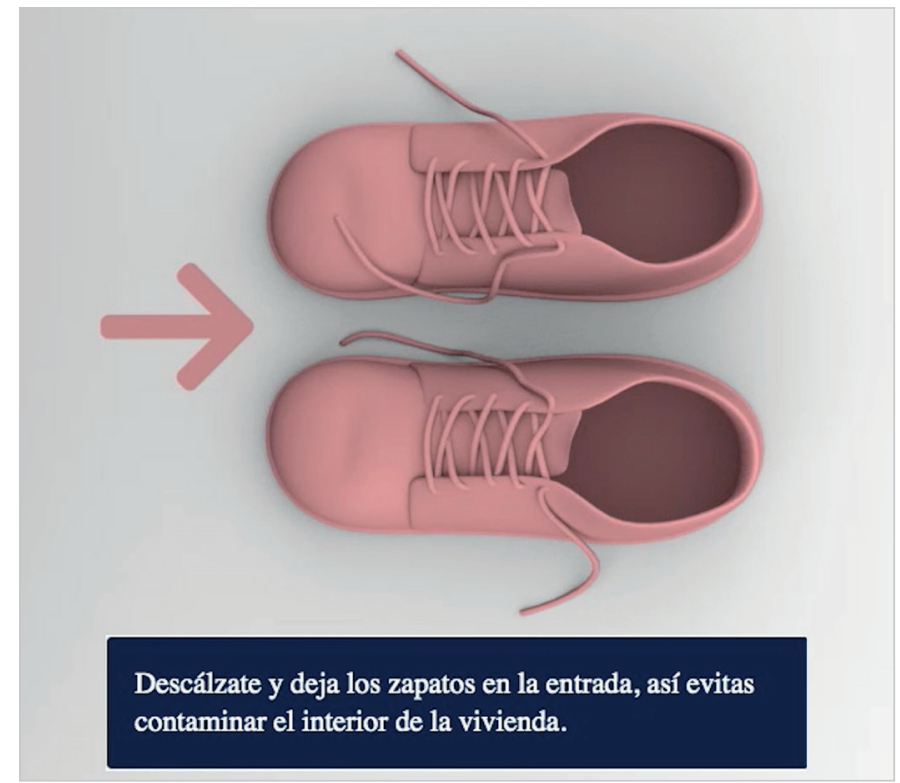

Imagen 1. Captura del reportaje multimedia de La Vanguardia 
En un reciente estudio de Reuters Institute (2020) se detectaba que los ciudadanos españoles son los que menos consideran que el Gobierno les ha ayudado a entender la pandemia (40\%). Coincide, además, que son quienes más responsabilidad atribuyen a los políticos en la diseminación de falsedades sobre el coronavirus.

\subsubsection{Nuevos medios para informar sobre el Covid-19}

Mientras la Comisión Europea adoptaba el 8 de abril una recomendación sobre el uso de los medios digitales como instrumento importante para las medidas de contención ${ }^{2}$ y varios países y ciudades empleaban varias tecnologías de última generación para combatir las consecuencias de la pandemia provocada por el Covid-19 (robots desinfectantes, cascos inteligentes, drones equipados con cámaras térmicas y software de reconocimiento facial, geolocalización...), los medios digitales, tanto nativos como matriciales aprovecharon las herramientas más sofisticadas que incorporaron en los últimos tiempos para una mejor cobertura informativa.

Además de los programas de verificación, algunos medios han puesto en marcha iniciativas que resultan especialmente uitiles para la información de calidad en tiempos de bulos y de estrategias desde distintos ámbitos para alimentar la desinformación sobre cuestiones de salud. Nos referimos a la cobertura con drones para fotos o la creación de un chatbot para informar sobre dudas en relación con el Covid-19. Varios medios han insertado uno que destacó desde el principio, Carina, que es un chatbot conversacional en español sobre coronavirus basado en inteligencia artificial, creado sobre la plataforma de Google Cloud, y que utiliza fuentes de la Organización Mundial de la Salud y fuentes gubernamentales.

Las aplicaciones de búsqueda y gestión de datos, de visualización dinámica de la información y de inteligencia artificial han formado parte del paquete de "alta tecnología" que han empleado los medios digitales para mejorar la cobertura de la información de las consecuencias del Covid-19 para la sociedad. Los medios nativos digitales y los matriciales o migrantes digitales han focalizado sus recursos tecnológicos incorporados en los últimos tiempos a una mejor cobertura de la pandemia. Su focalización en las causas y efectos del Covid-19 en todos los sectores han animado a experimentar en piezas periodísticas para mejorar la calidad y la presentación.

La inteligencia artificial, que se ha utilizado como una herramienta para apoyar la lucha contra la pandemia viral en distintos ámbitos, ha mostrado sus fortalezas para la creación de prospecciones para piezas periodísticas, buena parte de ellas presentadas luego mediante infografías o piezas multimedia. Los datos y el periodismo de datos han permitido contar otras perspectivas, más documentadas y con relatos más argumentados a partir de gestión de bases y de estadísticas de prospección. La disponibilidad de datos a gran escala ha propiciado la creación de piezas con estructuras y formatos actualizados.

La geolocalización de móviles ha estado de actualidad en distintos tratamientos informativos, sobre todo porque se ha empleado para el seguimiento del confinamiento durante el estado de alarma, mientras algunas herramientas se han mostrado especialmente útiles para los periodistas digitales a la hora de contar historias, como los gráficos con Datawrapper (en Eldiario.es, Elconfidencial.com, Lavozdegalicia.es...), o Flourish, que emplean The guardian (theguardian.com) o Financial times (ft.com), o Esri para los mapas (Lavanguardia.es).

Al tiempo que los medios digitales empleaban programas de última generación para la cobertura informativa y para la elaboración de piezas periodísticas multimedia, algunas organizaciones han creado webs o servicios especiales con consejos y ayudas para los periodistas. Las principales redes de periodismo como la Internacional Journalists' Network (IJnet), la Global Investigative Journalism Network (GIJN) y el International Consortium of Investigative Journalists (ICIJ) han creado webs especiales, han aportado recursos y los han puesto en libre acceso, al tiempo que entidades y observatorios han creado servicios útiles de apoyo a los periodistas (ver tabla 2).

Tabla 2. Recursos para periodistas ante la cobertura del Covid-19

\begin{tabular}{|l|l|}
\hline \multicolumn{1}{|c|}{ Organización } & \multicolumn{1}{c|}{ Localización } \\
\hline Ijnet & https://ijnet.org/en/resource/covid-19-resources-journalists \\
\hline Gijn & https://gijn.org/2020/03/10/tips-for-journalists-covering-covid-19 \\
\hline Icij & https://www.icij.org/tags/covid-19 \\
\hline Poynter & https://www.poynter.org/reporting-editing/2020/how-to-move-beyond-scary-covid-19-stories \\
\hline Dart Center & https://dartcenter.org/resources/covering-coronavirus-resources-journalists \\
\hline Journalist's resource & https://journalistsresource.org/studies/society/public-health/covid-19-coronavirus-epidemiology \\
\hline Ethical Journalism Network & https://ethicaljournalismnetwork.org/media-ethics-safety-and-mental-health-reporting-in-the-time-of-covid-19 \\
\hline
\end{tabular}

\section{Discusión y conclusiones}

La contraposición entre lecciones previas y situación reciente arroja como primera conclusión la desatención a las pautas y recomendaciones extraídas de los análisis sobre comunicación en crisis de salud pública anteriores. A pesar de que no hay dos situaciones idénticas y que cada crisis presenta una serie de características particulares, sí hay patrones de conducta identificados, tanto desde el punto de vista de la comunicación institucional como de la cobertura de prensa. 
Respecto de las lecciones previas sobre comunicación institucional, puede extraerse que se incumplieron aspectos relativos a la preparación de la comunidad para el escenario más negativo, se sobreprotegió con un mensaje de calma en etapas iniciales, que supuso luego un choque con la realidad y con la nueva comunicación efectuada. De la calma a la tempestad, sin preparación para una transición más gradual. Es ya bajo la tempestad, cuando se activan los mensajes de alerta, de responsabilidad individual y colectiva o de autoeficacia.

La fórmula y discurso de portavocía aplicada resulta contraria a las recomendaciones de comunicación de riesgos y de crisis. De hecho, supone una novedad respecto a situaciones anteriores, por la elevada cantidad de voces oficiales. Una portavocía técnica e institucional multiplicada permite, en positivo, llevar la iniciativa y ser proactivos en los mensajes enviados a la sociedad. En negativo, multiplica los riesgos de contradicción del mensaje y copa todo el espacio mediático, lo satura, abriendo para la ciudadanía un escenario de atención y de tensión permanente.

Dicha sobreexposición del discurso institucional no correlaciona necesariamente con la sensación de una mayor transparencia. La crítica corporativa de los periodistas al sistema de preguntas de las conferencias de prensa arroja sombras, a pesar de esa doble cita diaria de intervenciones ante los medios. La poca profundización en los datos facilitados (que son cifras de conjunto y que no bajan al detalle, por ejemplo) y su grado de adecuación a la realidad han sido otros de los aspectos informativos cuestionados. La incertidumbre no debe estar reñida con la transparencia. En este sentido, Covello (2010) remarca la necesaria incertidumbre que debe impregnar el mensaje de los agentes de salud pública como factor que acrecienta además su credibilidad y confianza.

En cuanto a la cobertura de prensa, el foco de atención ha sido exponencial conforme los casos se incrementaban. Se ha realizado un importante esfuerzo de cobertura informativa incorporando canales para la fidelización (newsletter o podcasts), además de establecer en portada una sección fija dedicada a los datos y evolución de la pandemia actualizada diariamente (ver imagen 2).

Crisis Covid-19

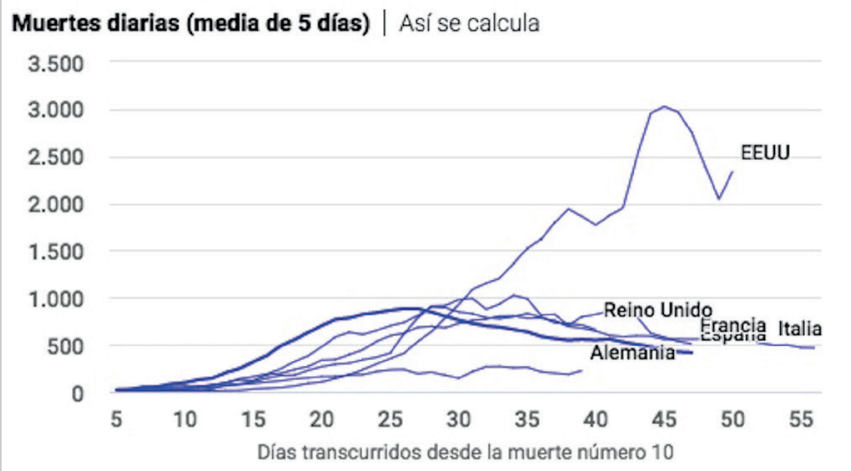

Newsletter | En imágenes | Datos actualizados | Curvas | Guía ciudadana | El virus | • Directo

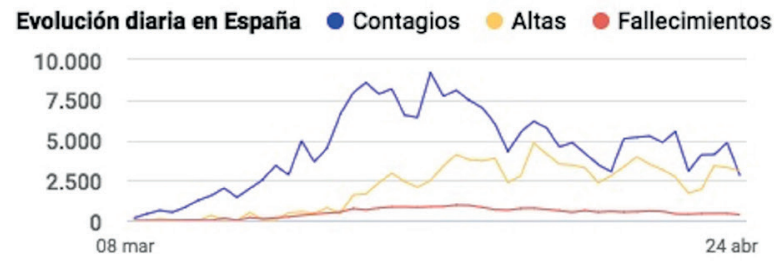

Por CCAA· Total de positivos y crecimiento diario

Madrid

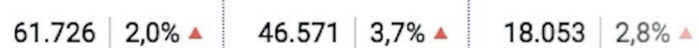

Imagen 2. Espacio fijo en portada actualizable sobre Covid-19 en Elconfidencial.com.

El alarmismo detectado en coberturas periodísticas previas también ha estado presente. Se hereda el lenguaje bélico de la comunicación institucional, que lo utiliza para activar la alerta, la urgencia en la adopción de la norma (con todas las modificaciones que comporta) y el sentimiento de unión interna y activación, pero que, al mismo tiempo, inevitablemente, provoca miedo a nivel social. No hay guerra, sino un tiempo de guerra (Xifra, 2020). Las muertes como indicador diario de la evolución local de la pandemia también funcionan en este sentido.

"La muerte se considera el factor que contribuye más al miedo en cualquier crisis de salud pública" (Khishnatary; Gadekar, 2014, p. 731).

La Asociación Española de Comunicación Científica (AECC, 2020) explica que es necesario que la opinión pública conozca los hechos relevantes sobre esta crisis sanitaria y que los medios de comunicación aborden un tema tan sensible evitando contagiar en la audiencia el alarmismo y el miedo. Además, debe procurarse un enfoque periodístico humano y que no caiga en el sensacionalismo a la hora de relatar las historias que están detrás de la cifra. 
Los datos numéricos se han convertido en el elemento estrella y los infográficos, en correlación, en el componente gráfico más importante en las piezas periodísticas a nivel nacional e internacional, en medios nativos digitales o matriciales. Ello ha conllevado una apuesta por programas novedosos de gestión de datos y visualización interactiva. Las piezas periodísticas multimedia y divulgativas (no centradas en las estadísticas) han sido menos prolíficas.

También los esfuerzos han sido evidentes en la lucha contra las fake news. El escenario de la emergencia es especialmente proclive para la difusión de datos sesgados y distorsionados por el impacto de las redes sociales (Mayo-Cubero, 2020). La desinformación se ha convertido, estas semanas, en un fenómeno fuertemente vinculado a la extensión del Covid-19 (Casero, 2020). Los bulos se expanden con facilidad a través de las redes sociales y los medios intensifican la lucha con herramientas de fact check creadas en los 2-3 últimos años (bien propias o en alianza con empresas de verificación de datos), centradas ahora en desinformaciones vinculadas a la pandemia.

En este sentido, las organizaciones, redes y observatorios de periodismo han publicado guías y recursos para ayudar a los periodistas a enfrentarse a la cobertura informativa del Covid-19, tanto desde el punto de vista de su seguridad personal, como de las fuentes de información más adecuadas, la perspectiva ética o recomendaciones de cara al tratamiento informativo. En previsión de futuras crisis, los periodistas deberían mejorar la preparación y capacidad de respuesta proporcionando una cobertura que combine diferentes tipos de información y evite presentar la información de riesgos en ausencia del consejo de cómo los individuos deben actuar (Ophir, 2019).

"El relato informativo en las crisis debería ser capaz de ofrecer información que salve vidas y minimice daños en el corto plazo. En el medio y en el largo plazo, debería también ayudar a la comunidad a recuperarse de la tragedia y a mejorar la capacidad de resiliencia ante crisis futuras. Y ese relato informativo sólo puede construirse desde el equilibrio entre el rigor y el interés humano, entre el uso ecuánime de las fuentes oficiales y las no oficiales" (Mayo-Cubero, 2020, p. 9).

La presente crisis sanitaria ha supuesto una realidad mucho más compleja y grave que las precedentes ya citadas, como la del ébola o la gripe A, más acotadas en su duración temporal y en los daños ocasionados. Existe una enorme responsabilidad en la comunicación con la ciudadanía en una situación como la presente en todos los actores del sistema comunicativo, especialmente, los actores institucionales y los medios de comunicación. Los primeros afrontan el reto de la transparencia, la coherencia, el entendimiento con los distintos stakeholders, el liderazgo y la divulgación. Los segundos, el de informar en profundidad desde un punto de vista crítico, contrastando la información oficial, pero colaborando en la difusión de los mensajes, aportando el lado humano sin sensacionalismo ni alarmismo.

La gestión de la comunicación e información a la sociedad debe tomar nota de las lecciones aprendidas en situaciones previas y planificar y entrenar el afrontamiento de crisis de salud pública para garantizar que, en nuevos escenarios similares, haya una respuesta comunicativa preparada y fundamentada en el conocimiento adquirido. La Organización Mundial de la Salud (2015), en el Marco de Sendai para la Reducción del Riesgo de Desastres 2015-2030, hablaba de la necesaria cultura de la prevención.

Se recomienda la continuación de esta línea de investigación dada la importancia del estudio detallado de la presente crisis de salud pública de cara a futuras crisis o próximas oleadas de esta misma crisis.

La gestión de la comunicación y la cobertura de prensa deben tomar nota de las lecciones aprendidas

\section{Notas}

1. Puede accederse a MoMo en el siguiente enlace:

https://momo.isciii.es/public/momo/dashboard/momo_dashboard.html\#nacional

2. El texto de la recomendación puede consultarse en: https://ec.europa.eu/info/files/recommendation-apps-contact-tracing_en

\section{Referencias}

Asociación Española de Comunicación Científica (2020). Consejos para informar sobre el nuevo coronavirus. Madrid: AECC.

https://cutt.ly/UyyBEw5

Beck, Ulrich (2002). La sociedad del riesgo global. Madrid: Siglo XXI. ISBN: 9788432312618.

Brennan, Bryna (2014). Una revisión general sobre comunicación de riesgos. Pan American Health Organization. https://cutt.ly/tyyMglk 
Calleja-Reina, María-Antonia; Becerra, Elena (2017). “La comunicación institucional del virus del ébola en España: análisis de la gestión estratégica online y offline de la crisis". In: Terrón, José Luis; Peñafiel, Carmen; Catalán, Daniel. Avances en investigación y buenas prácticas en comunicación y salud, pp. 35-48. La Laguna: Sociedad Latina de Comunicación Social. ISBN: 9788416458806

Casero-Ripollés, Andreu (2020). "Impact of Covid-19 on the media system. Communicative and democratic consequences of news consumption during the outbreak". El profesional de la información, v. 29, n. 2, e290223.

https://doi.org/10.3145/epi.2020.mar.23

CDC (2020). Pandemia H1N1 del 2009 (virus H1N1pdm09).

https://espanol.cdc.gov/flu/pandemic-resources/2009-h1n1-pandemic.html

Centro de Estudios de Ciencia, Comunicación y Sociedad (2014). Informe Quiral 2014. Fundació Vila Casas. https://www.fundaciovilacasas.com/es/informe-quiral

Churchill, Elliot (2000). "Relaciones efectivas con los medios". In: Eric K. Noji (ed.). Impacto de los desastres en la salud pública, pp. 122-133. Bogotá: Pan American Health Organization. ISBN: 0195095707

Covello, Vincent (2001). "Risk communication, radiation, and radiological emergencies: strategies, tools, and techniques". Health physics, v. 101, n. 5, pp. 511-530.

https://doi.org/10.1097/HP.0b013e3182299549

Covello, Vincent (2010). H1N1 risk and crisis communication: successes and challenges. Atlanta: Center for Disease Control and Prevention.

https://slideplayer.com/slide/4520533

DeGiusti, Maria; Mannocci, Alice; Miccoli, Silvia; Palazzo, Caterina; Di Thiene, Domitilla; Scalmato, Valeria; Ursillo, Paolo; Monteduro, Maria-Antonietta; Turri, Alberto; Mazzoli, Pier-Giovanni; La Torre, Giuseppe (2012). "Risk communication during health crises: results of a cross-sectional study to evaluate the effectiveness of adopted corporate communication strategies during the H1N1 influenza pandemic in Italy and on the training needs of health professionals". Igiene e sanità pubblica, v. 68, n. 1, pp. 69-84.

https://www.ncbi.nlm.nih.gov/pubmed/22507993

Ding, Huiling (2014). "Transnational quarantine rhetorics: Public mobilization in SARS and in H1N1 flu". Journal of medical humanities, v. 35, n. 2, pp. 191-210.

https://doi.org/10.1007/s10912-014-9282-8

ECDC (2013). A literature review on effective risk communication for the prevention and control of communicable diseases in Europe. Stockholm: ECDC. ISBN: 9789291933952.

Esparza, José (2016). “Epidemias y pandemias virales emergentes: ¿Cuál será la próxima?” Investigación clínica, v. 57, n. 3, pp. 231-235.

http://ve.scielo.org/scielo.php?script=sci_arttext\&pid=S0535-51332016000300001

Frisby, Brandi N.; Veil, Shari R.; Sellnow, Timothy L. (2014). "Instructional messages during health-related crises: Essential content for self-protection". Health communication, v. 29, n. 4, pp. 347-354.

https://doi.org/10.1080/10410236.2012.755604

Gadekar, Rahul; Krishnatray, Pradeep; Ang, Peng H. (2014). "Framing of the H1N1 flu in an Indian newspaper". Journal of creative communications, v. 9, n. 1, pp. 49-66.

https://doi.org/10.1177/0973258613517438

García-Frontiñán, Eva (2017). Comunicación de crisis en los gabinetes de prensa sanitarios. Análisis de la producción periodística y de la información publicada sobre la gripe A. Madrid: Universidad Complutense de Madrid.

https://eprints.ucm.es/41935

Gérvas, Juan; Hernández-Aguado, Ildefonso (2009). “Aciertos y errores en la gestión de las crisis de salud pública en España". Gaceta sanitaria, v. 23, n. 1, pp. 67-71.

https://doi.org/10.1016/j.gaceta.2007.11.001

Gou-Núñez, Míriam (2017). Crisis de salud en los medios de comunicación: El ébola en España. Análisis de los diarios La Vanguardia, El Mundo y El País. Barcelona: Universidad Autónoma de Barcelona.

https://ddd.uab.cat/record/180517

Jang, Kyungeun; Park, Namkee (2018). "The effects of repetitive information communication through multiple channels on prevention behavior during the 2015 MERS outbreak in South Korea". Journal of health communication, v. 23, n. 7, pp. 670-678.

https://doi.org/10.1080/10810730.2018.1501440 
Jones, Sandra C.; Waters, Louise; Holland, Omnia; Bevins, John; Iverson, Don (2010). “Developing pandemic communication strategies: Preparation without panic". Journal of business research, v. 63, n. 2, pp. 126-132. https://doi.org/10.1016/j.jbusres.2009.02.009

Jurado-Salván, Enrique; Jurado-Izquierdo Marta (2014). “Los errores de comunicación en la crisis del ébola”. Cuadernos de periodistas: revista de la Asociación de la Prensa de Madrid, n. 29, pp. 90-99.

http://www.cuadernosdeperiodistas.com/los-errores-de-comunicacion-en-la-crisis-del-ebola

Krishnatray, Pradeep; Gadekar, Rahul (2014). "Construction of death in H1N1 news in The Times of India”. Journalism, v. 15, n. 6, pp. 731-753.

https://doi.org/10.1177/1464884913496497

Lorente, José-Ignacio (2010). H1N1. "Virus, discurso del riesgo y gestión mediática de la alarma sanitaria". In: Comunicación y desarrollo en la era digital. Il Congreso internacional de la Asociación Española de Investigadores de la Comunicación (AE-IC). Málaga: AEIC.

Mahy, Patrick; Collard, Jean-Marc; Gala, Jean-Luc; Herman, Philippe; De-Groof, Dirk; Quoilin, Sophie; Sneyers, Myriam (2017). "Health crises due to infectious and communicable diseases: European preparedness and response tools in an international context". Journal of business continuity \& emergency planning, v. 10, n. 4, pp. 353-366.

https://hstalks.com/article/340/health-crises-due-to-infectious-and-communicable-d

Mayo-Cubero, Marcos (2020). "News sections, journalists and information sources in the journalistic coverage of crises and emergencies in Spain". El profesional de la información, v. 29, n. 2, e290211.

https://doi.org/10.3145/epi.2020.mar.11

Mestre-Ortega, Pilar; Ruiz-de-Gordoa, Juan-José; Mira-Roldán, Amparo; Buendía-Domínguez, Rocío; Bravo-Hernández, Miguel-Ángel (2018). "Análisis de impactos de los medios de comunicación de los casos de la fiebre hemorrágica de Crimea-Congo en la Comunidad de Madrid (2016). El papel de la comunicación institucional en crisis de salud pública". Revista de comunicación y salud, v. 8, n. 1, pp. 99-109.

https://doi.org/10.35669/revistadecomunicacionysalud.2018.8(1).99-109

Ministerio de Sanidad (2020). "Situación actual". https://cutt.ly/dyofqrp

Mondragón, Nahia (2016). “Redes sociales en tiempos de riesgo: Analizando el ébola mediante Twitter”. Opción: Revista de ciencias humanas y sociales, n. 11, pp. 740-756.

Monjas-Eleta, María; Gil-Torres, Alicia (2017). “Comunicación institucional y tratamiento periodístico de la crisis del ébola en España entre el 6 y el 8 de octubre de 2014". Revista de comunicación, v. 16, n. 1, pp. 97-121.

https://revistadecomunicacion.com/article/view/1009

Ophir, Yotam (2019). "The effects of news coverage of epidemics on public support for and compliance with the CDC An experimental study". Journal of health communication, v. 24, n. 5, pp. 547-558.

https://doi.org/10.1080/10810730.2019.1632990

Organización Mundial de la Salud (2015). Marco de Sendai para la Reducción del Riesgo de Desastres 2015-2030. WHO. https://www.unisdr.org/files/43291_spanishsendaiframeworkfordisasterri.pdf

Park, Sejin; Boatwright, Brandon; Avery, Elizabeth-Johnson (2019). "Information channel preference in health crisis: Exploring the roles of perceived risk, preparedness, knowledge, and intent to follow directives". Public relations review, v. 45, n. 5 .

https://doi.org/10.1016/j.pubrev.2019.05.015

Qiu, Wuqi; Chu, Cordia (2019). "Clarification of the concept of risk communication and its role in public health crisis management in China". Disaster medicine and public health preparedness, v. 13, n. 5-6, pp. 834-836.

https://doi.org/10.1017/dmp.2019.10

Qiu, Wuqi; Chu, Cordia; Hou, Xiaohui; Rutherford, Shannon; Zhu, Bin; Tong, Zhendong; Mao, Ayan (2018). "A comparison of China's risk communication in response to SARS and H7N9 using principles drawn from international practice". Disaster medicine and public health preparedness, v. 12, n. 5, pp. 587-598.

https://doi.org/10.1017/dmp.2017.114

Reuters Institute (2020). Navigating the 'infodemic': how people in six countries access and rate news and information about coronavirus. Oxford: Reuters Institute for the Study of Journalism.

https://cutt.ly/ZyillSy

Reynolds, Barbara; Seeger, Matthew W. (2005). "Crisis and emergency risk communication as an integrative model". Journal of health communication, v. 10, n. 1, pp. 43-55.

https://doi.org/10.1080/10810730590904571 
Rodríguez-Andrés, Roberto (2011). "La efectividad del uso del miedo como factor persuasivo en la comunicación de riesgos en las crisis sanitarias”. Revista de comunicación y salud, v. 1, n. 2, pp. 33-46.

https://doi.org/10.35669/revistadecomunicacionysalud.2011.1(2).33-46

Rossmann, Constance; Meyer, Lisa; Schulz, Peter J. (2018). "The mediated amplification of a crisis: Communicating the A/H1N1 pandemic in press releases and press coverage in Europe". Risk analysis, v. 38, n. 2, pp. 357-375. https://doi.org/10.1111/risa.12841

Sandman, Peter M. (2012). Responding to community outrage: Strategies for effective risk communication. American Industrial Hygiene Association. ISBN: $093262751 \mathrm{X}$

Savoia, Elena; Lin, Leesa; Viswanath, Kasisomayajula (2013). Communications in public health emergency preparedness: a systematic review of the literature. Biosecurity and bioterrorism: biodefense strategy, practice, and science, v. 11 , n. 3, pp. 170-184.

https://doi.org/10.1089/bsp.2013.0038

Scarcella, Carmelo; Antonelli, Laura; Orizio, Grazia; Rossmann, Costance; Ziegler, Lena; Meyer, Lisa; García-Jiménez, Leonarda; Losada, José-Carlos; Correia, João; Soares, Joana; Covolo, Loredana; Lirangi, Enrico; Gelatti, Umberto (2013). "Crisis communication in the area of risk management: the CriCoRM project". Journal of public health research, v. 2, n. 2, pp. 118-121.

https://doi.org/10.4081/jphr.2013.e20

Smith, Katherine C.; Rimal, Rajiv N.; Sandberg, Helena; Storey, John D.; Lagasse, Lisa; Maulsby, Catherine; Rhoades, Elizabeth; Barnett, Daniel J.; Omer, Saad B.; Links, Jonathan M. (2013). “Understanding newsworthiness of an emerging pandemic: International newspaper coverage of the H1N1 outbreak". Influenza and other respiratory viruses, v. 7, n. 5, pp. 847-853.

https://doi.org/10.1111/irv.12073

Takahashi, Bruno; Tandoc Jr, Edson C.; Carmichael, Christine (2015). "Communicating on Twitter during a disaster: An analysis of tweets during Typhoon Haiyan in the Philippines". Computers in human behavior, n. 50, pp. 392-398. https://doi.org/10.1016/j.chb.2015.04.020

Vos, Sarah C.; Buckner, Marjorie M. (2016). "Social media messages in an emerging health crisis: Tweeting bird flu". Journal of health communication, v. 21, n. 3, pp. 301-308. https://doi.org/10.1080/10810730.2015.1064495

World Health Organization (2004). Sixth futures forum on crisis communication. ISBN: 9289010673 http://www.euro.who.int/document/E85056.pdf

World Health Organization (2005). Outbreak communication guidelines. https://www.who.int/csr/resources/publications/WHO_CDS_2005_28en.pdf

World Health Organization (2008). World Health Organization outbreak communication planning guide. WHO. ISBN: 978 9241597449 https://www.who.int/ihr/elibrary/WHOOutbreakCommsPlanngGuide.pdf

World Health Organization (2013). Health and environment: communicationg the risks. ISBN: 9789289000512 http://www.euro.who.int/_data/assets/pdf file/0011/233759/e96930.pdf

World Health Organization (2020). Risk communication and community engagement readiness and response to coronavirus disease (Covid-19). WHO.

https://www.who.int/publications-detail/risk-communication-and-community-engagement-readiness-and-initialresponse-for-novel-coronaviruses-(-ncov)

Xifra, Jordi (2020). “Comunicación corporativa, relaciones públicas y gestión del riesgo reputacional en tiempos del Covid-19". El profesional de la información, v. 29, n. 2, e290220.

https://doi.org/10.3145/epi.2020.mar.20

Yang, Sung-Un (2018). Effects of government dialogic competency: The MERS outbreak and implications for public health crises and political legitimacy. Journalism and mass communication quarterly, v. 95, n. 4, pp. 1011-1032. https://doi.org/10.1177/1077699017750360 